\title{
Efficient storage of microCT data preserving bone morphometry assessment
}

\author{
Joan Bartrina-Rapesta, Francesc Aulí-Llinàs, Ian Blanes, Michael W. Marcellin, \\ Victor Sanchez, and Joan Serra-Sagristà \\ Joan Bartrina-Rapesta, Francesc Auli-Llinàs, Ian Blanes, and Joan Serra-Sagristà are with the Department \\ of Information and Communications Engineering, Universitat Autònoma de Barcelona, Bellaterra, Spain. \\ Michael W. Marcellin is with the Department of Electrical and Computer Engineering, University of \\ Arizona, Tucson, USA. \\ Victor Sanchez is with the Department of Computer Science, University of Warwick, Coventry, UK. \\ (Corresponding author e-mail: joan.bartrina@uab.cat).
}

\begin{abstract}
Preclinical micro-computed tomography (microCT) images are of utility for 3D morphological bone evaluation, which is of great interest in cancer detection and treatment development. This work introduces a compression strategy for microCTs that allocates specific substances in different Volumes of Interest (VoIs). The allocation procedure is conducted by the Hounsfield (HU) scale. The VoIs are coded independently and then grouped in a single DICOM-compliant file. The main advantages of the proposed method are that it permits the use of different codecs, it can identify and transmit data corresponding to a particular substance in the compressed domain without decoding the volume(s), and allows the computation of the 3D morphometry without needing to store or transmit the whole image. Experimental results indicate that the proposed approach reduces the transmitted data in more than $90 \%$ when the 3D morphometry evaluation is performed for common substances such as high density and low density bone. Results also indicate that JPEG2000 is the most convenient codec in such a coding scheme. This work can be easily extended to other imaging modalities and applications that work with the HU scale.
\end{abstract}

Keywords:

Tomography, 3D morphometry, medical image compression, DICOM, JPEG2000, H.264, JPEG-LS 


\section{Introduction}

Human breast, lung, and prostate cancer can induce bone metastasis, which affects the bone structure [1]. To better understand the interactions between these diseases and the bone structure during the development of metastasis, preclinical researchers develop models using small living animals. These models help to evaluate new therapeutic approaches [2, 3]. The use of micro-computed tomography (microCT) images has become a common approach to assess structural changes in the bones of animals by means of 3D morphometry [4, 5, 6].

During the microCT scanning process, imaging data belonging to air, fat, muscle, bone, bed, and other substances are captured. The reconstruction of the whole volume of the animal has high computational costs due to the large amount of data involved. So, rather than reconstructing and storing the whole volume, in practice, only a relevant volume (RV) that contains a portion of the whole data is reconstructed, stored, and used for the 3D morphometry evaluation.

To obtain qualitative and quantitative bone morphometric measurements, specialists segment the bone tissue from the rest of the data within an RV through a thresholding procedure. This procedure is required because RVs may contain many data that do not correspond to the bone tissues. These data are not necessary for the 3D morphometry computation. The 3D morphometry is computed solely using the bone data [6, 7]. Research institutions store sets of microCT data in servers to allow their access and distribution to other institutions via the Internet. The Digital Imaging and Communications in Medicine (DICOM) standard [8] is typically adopted to store and distribute such medical images [9]. This standard includes coding (i.e., compression) systems to reduce the size of the data stored. The most relevant coding systems supported in DICOM are Lempel-Ziv-Welch (LZW) [10], JPEG [11], JPEG lossless (JPEG-LS) [12], JPEG2000 [13], and H.264 [14].

Progressive lossy-to-lossless coding of medical images has been widely studied in the literature. Without aiming to be exhaustive, [15] presents a 3D coding technique based on discrete cosine transform and several proposals based on discrete wavelet transform. A modification of the set partition hierarchical trees (SPIHT) method for 
handling 3D medical data sets is introduced in [16]. An object extension of SPIHT is presented in [17]. Sanchez et al. [18] employed the anatomical symmetries of the human body in a prediction technique that employs the wavelet transform. A JPEG2000 region of interest (ROI) coding method that can prioritize multiple ROIs at different priorities guaranteeing lossy-to-lossless coding for the ROI and the BG is proposed in [19]. More recently, a region-based coding method that distinguishes three different types of regions, namely, ROI, non-ROI, and background is described in [20]. In this work, the ROI is coded with a coding system such as RLE, LZE, or ZIP, the non-ROI is coded with SPIHT, and the background is disregarded. Despite the advantages that these coding systems may provide, none of them is suited for professional medical scenarios. This is because most of them are not supported in DICOM, others can only be employed with a particular coding system, some do not recover the original image, and others need to carry out the ROI selection manually.

This paper introduces a novel DICOM-compliant coding strategy that uses the Hounsfield (HU) scale to identify different substances within an RV of a microCT image. These substances are allocated in different Volumes of Interest (VoIs) through a segmentation process performed prior to the data storage. The segmentation process allows the organization of an RV in different VoIs that are encoded independently and then grouped together in a single DICOM-compliant file. Each VoI contains a specific substance. This individual treatment of the substances allows their encoding using any technique included in DICOM. Also, it permits the identification and transmission of the data corresponding to a particular substance with neither decoding nor transmitting the others. This can be conceptually seen as scalability by substance, a new feature of the coding system. The proposed method also permits the computation of the $3 \mathrm{D}$ morphometry transmitting a reduced amount of data. To enhance the coding performance, an offset operation that compacts the dynamic range of the VoIs is performed. Extended experimental results analyzing coding performance and accuracy of the 3D morphometry validates the soundness of the proposed method.

The rest of this paper is structured as follows. Section 2 briefly describes materials and methods, Section 3 describes the proposed approach, and Section 4 provides results and discussions. The last section concludes this work with a brief summary. 
Table 1: HU unit ranges for different substances in microCT images, for the eXplore Locus microCT device.

\begin{tabular}{|r|c|r|}
\hline Substance & Acronym & HU units \\
\hline \hline Air & $\mathrm{A}$ & $<-1000$ \\
Ethyl Alcohol & $\mathrm{E}$ & {$[-1000,-700)$} \\
Fat & $\mathrm{F}$ & {$[-700,-150)$} \\
Water & $\mathrm{W}$ & {$[-150,700)$} \\
Low Density Bone & LB & {$[700,3000)$} \\
High Density Bone & HB & $\geq 3000$ \\
\hline
\end{tabular}

\section{Materials and Methods}

\subsection{MicroCT Acquisition}

The intensity values of microCT volumes are given in $\mathrm{HU}$ units. This allows the identification of different substances since they are allocated in non-overlapping ranges of intensity. Table 1 provides the HU scale included in the reference guide of the eXplore Locus device employed in this work (see [21]). The HU scale can be finely adjusted by the specialist thanks to a calibration object that is scanned along with the animal. Other CT images (that are not necessarily microCT) also have a HU scale associated, so our method may be employed for other types of CT images.

\subsection{MicroCT Analysis}

In general, the evaluation of bone structural changes produced by bone metastasis is carried out by computing the 3D morphometry using data corresponding to low-density bone (LB) and high-density bone (HB) [4, 6, 7, 22]. Let $B V, B S$, and $T V$ respectively denote the bone volume, the bone surface, and the tissue volume of an RV. The tissue volume corresponds to fat, water, low bone, and high bone. The statistics employed in 3D morphometry evaluation to analyze the cancellous bone are $V_{s}, S_{v}, T b . T h, T b . N$, and Tb.Sp. They respectively represent the bone volume fraction, the density of the 
bone for a given VoI, the average trabecular thickness, the number of trabeculae, and the average separation between trabeculae. They are obtained via

$$
\begin{gathered}
T b . T h=\frac{2-2 V_{v}}{S_{v}}, T b . N=\frac{2 V_{v}}{S_{v}} \text {, and } T b . S p=\frac{S_{v}}{2}, \\
\text { with } V_{v}=\frac{B V}{T V} \text { and } S_{v}=\frac{B S}{B V},
\end{gathered}
$$

with $V_{v}$ and $S_{v}$ being the bone volume ratio and the bone density for a given VoI, respectively. These architectural measures only depend on $B V, T V$, and $B S$ for a specific VoI. In the case of a lossless encoding and decoding of a VoI, $B V$ and $B S$ are calculated without error. $T V$ needs to be stored as auxiliary data for each VoI during the segmentation procedure. It is generally stored in the metadata headers of the DICOM files.

The 3D morphometry is computed using the boneJ software Library [23] with ImageJ [24]. To assess morphological differences, specialists commonly compute the 3D morphometry of bone between two different specimens, or between bone of the same specimen before and after an experimental treatment. It is considered that a difference below $2 \%$ is insignificant, between $10 \%$ and $18 \%$ is evident, and larger to $18 \%$ is significant [25].

\subsection{Materials}

The RVs employed in this work are provided by the Center for Animal Biotechnology and Gene Therapy (CBATEG) [26], from the Universitat Autònoma de Barcelona. The microCTs are acquired by the eXplore Locus microCT imaging device from General Electric. This device generates an RV as a collection of slices with a bit depth of 14 bps including sign at a voxel resolutions of 93,45 , or $27 \mu m$. Results reported in the experimental section are obtained with a corpus of $19 \mathrm{RVs}$ belonging to 4 different mice. They are acquired with a voxel resolution of $27 \mu \mathrm{m}$. Although $27 \mu \mathrm{m}$ might not be the most suitable resolution for bone evaluation since the microstructure of trabecular bone may be missing [27, 28], it is the highest resolution supported in this device and so it is used in CBATEG for bone morphometry. The characteristics of these RVs are reported in Table 2 
Table 2: RVs employed in this work. From left to right, the columns provide the body location, the RV identification, the lower-left corner and first slice of the RV within the volume, the dimensions of the RV, the size of file before coding (in megabytes (MB)), the min and max intensity value, the percentage of $\mathrm{HB}$ and LB, and the order-0 entropy.

\begin{tabular}{|c|c|c|c|c|c|c|c|c|c|}
\hline Location & RV\# & $(x, y, z)$ & $\left(R_{x}, R_{y}, R_{z}\right)$ & Size & $\min$ & $\max$ & $\%$ HB & $\%$ LB & Entropy \\
\hline & 1 & $(804,476,806)$ & $(510,375,475)$ & $174 \mathrm{MB}$ & -2164 & 5579 & 0.6 & 2.9 & 8.96 \\
& 2 & $(664,506,366)$ & $(420,505,735)$ & $298 \mathrm{MB}$ & -3003 & 5538 & 0.5 & 2.3 & 9.62 \\
& 3 & $(924,546,301)$ & $(445,440,575)$ & $215 \mathrm{MB}$ & -2634 & 5738 & 0.6 & 3.0 & 8.77 \\
& 4 & $(894,511,271)$ & $(515,510,715)$ & $359 \mathrm{MB}$ & -3644 & 5769 & 0.4 & 2.0 & 11.01 \\
& 5 & $(744,381,696)$ & $(420,400,405)$ & $130 \mathrm{MB}$ & -2214 & 5218 & 0.4 & 3.7 & 8.00 \\
& 6 & $(325,495,291)$ & $(874,456,840)$ & $258 \mathrm{MB}$ & -1216 & 1463 & 0.3 & 2.9 & 7.71 \\
\hline \multirow{5}{*}{ Hip 1 } & 1 & $(994,706,346)$ & $(325,375,390)$ & $91 \mathrm{MB}$ & -1216 & 1463 & 0.0 & 0.4 & 5.35 \\
& 2 & $(784,376,6)$ & $(510,525,590)$ & $302 \mathrm{MB}$ & -1513 & 7091 & 2.6 & 7.1 & 10.86 \\
& 3 & $(804,321,106)$ & $(410,430,575)$ & $194 \mathrm{MB}$ & -1529 & 2673 & 0.0 & 3.6 & 7.67 \\
& 4 & $(784,446,821)$ & $(555,500,170)$ & $90 \mathrm{MB}$ & -1494 & 4651 & 0.3 & 5.7 & 10.15 \\
& 5 & $(1079,671,146)$ & $(255,565,950)$ & $262 \mathrm{MB}$ & -1235 & 1824 & 0.0 & 0.8 & 9.45 \\
& 6 & $(654,716,771)$ & $(900,555,270)$ & $258 \mathrm{MB}$ & -1992 & 5120 & 0.1 & 4.7 & 9.82 \\
\hline Head 1 & 1 & $(929,256,81)$ & $(370,555,550)$ & $216 \mathrm{MB}$ & -2676 & 7593 & 2.3 & 8.3 & 8.92 \\
\hline & 1 & $(1024,586,236)$ & $(235,170,505)$ & $39 \mathrm{MB}$ & -2422 & 7035 & 5.4 & 13.0 & 8.16 \\
& 2 & $(1029,521,171)$ & $(285,415,655)$ & $148 \mathrm{MB}$ & -2191 & 7125 & 4.2 & 11.2 & 10.24 \\
& 3 & $(879,366,296)$ & $(495,440,680)$ & $283 \mathrm{MB}$ & -2422 & 8523 & 4.1 & 8.3 & 10.28 \\
& 4 & $(854,351,1046)$ & $(605,445,355)$ & $183 \mathrm{MB}$ & -2269 & 6297 & 0.8 & 4.4 & 10.25 \\
& 5 & $(1219,566,736)$ & $(295,540,585)$ & $178 \mathrm{MB}$ & -2245 & 6583 & 1.5 & 6.3 & 11.35 \\
& 6 & $(769,616,556)$ & $(830,415,325)$ & $214 \mathrm{MB}$ & -2480 & 7204 & 1.7 & 4.9 & 8.49 \\
\hline
\end{tabular}

\subsection{DICOM-compliant coding techniques}

As previously mentioned, the DICOM standard supports the coding systems RLE, LZW, JPEG, JPEG-LS, JPEG2000, and H.264. RLE does not provide competitive performance for CT image data and JPEG does not support lossless compression, so they are not employed herein. The remaining systems support lossless compression and achieve competitive performance. LZW is a dictionary-based technique that replaces strings of characters with single codes. JPEG-LS is based on the LOCO-I algorithm [29]. It has two coding stages, a predictor and an encoder. JPEG2000 is wavelet-based and features important functionalities such as progressive lossy-tolossless coding, spatial and quality scalability, random codestream access, and support for up to 38-bits of signed and unsigned data with up to 16384 slices. These features 
are not included in any other coding systems compliant with DICOM. H.264 is commonly employed for coding and distributing video content. It is based on block motion estimation and compensation. The extensions of the H.264 standard include the Scalable Video Coding (SVC), which enhances the interactive transmission (though such extension is not included in DICOM).

\section{Proposed Storage Approach}

The proposed method introduces a novel allocation procedure for microCT that allows the storage and identification of relevant substances in the compressed domain. First, a segmentation process that organizes an RV in different VoIs is applied. Each VoI contains a specific substance. These VoIs are then separately encoded. After encoding, they are grouped in a DICOM-compliant file. This individual treatment of the VoIs permits to encode them with different coding systems, identify and transmit the data corresponding to a particular substance (i.e., providing substance scalability), and compute the 3D morphometry without transmitting or decoding the whole RV.

The proposed scheme is illustrated in Figure 1. It has a pre-coding stage named "RV Allocation by Substances", a DICOM-compliant coder and decoder, and an (optional) post-decoding stage named "RV Reallocation". The "RV Allocation by Substances" and the "RV Reallocation" steps are highlighted in gray in the figure. They are described in the following section.

\subsection{RV Allocation by Substances}

As previously stated, a typical microCT image contains different substances of the scanned mouse and the surrounding environment. The most common substances are those reported in Table 1 . Since the ranges of the intensities for these substances do not overlap, the specialists carry out the distinction among the different substances of the image via a thresholding procedure that utilizes the $\mathrm{HU}$ value ranges associated to each substance [7, 22, 30]. The threshold may vary depending on the scanning parameters and environment conditions, though the specialists can adapt such thresholds according to the device and the calibration object. 

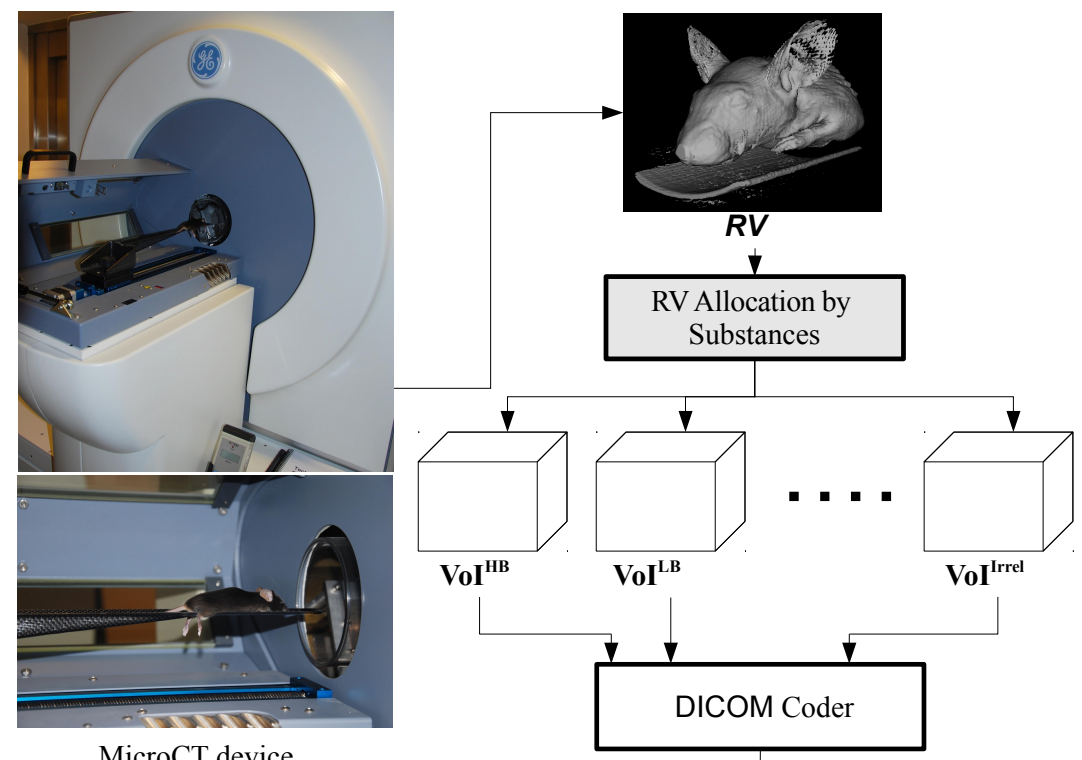

MicroCT device

DICOM-Compliant file

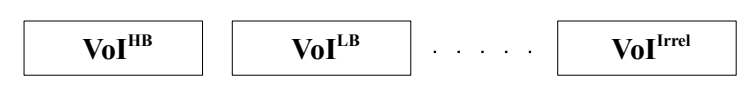

DICOM Decoder
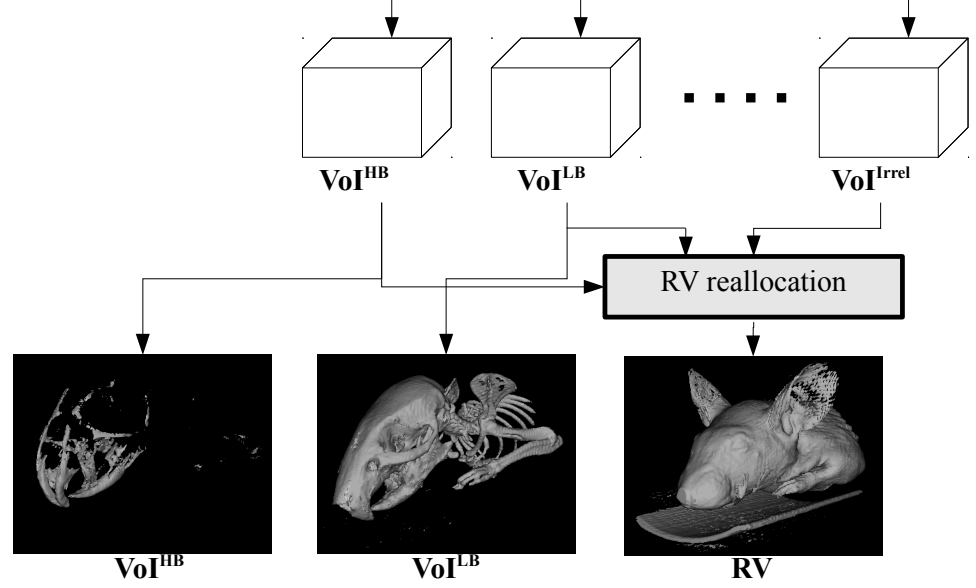

Figure 1: Illustration of the proposed coding scheme for microCT images. 
The goal of the "RV Allocation by Substances" procedure is to extract the relevant substances (generally, the HB and LB) from the original RV and copy them to independent VoIs. The remaining substances are considered irrelevant and are copied to another VoI. More precisely, the "RV Allocation by Substances" procedure takes as input an RV, with a dimension of $X$ rows, $Y$ columns, and $Z$ slices. The intensity of a sample within this volume is denoted by $\mathrm{RV}_{x, y, z}$, with the subindices $x, y, z$ indicating the row, column, and slice of the sample. Let $\mathrm{S}$ denote a biological substance and $\mathrm{VoI}^{\mathrm{S}}$ the VoI associated with such substance after the allocation procedure. The size of $\mathrm{VoI}^{\mathrm{S}}$ is identical to the original $\mathrm{RV}$, with the intensities of those samples corresponding to $\mathrm{S}$ being equal as in the original RV. The remaining samples are zeroes. An individual VoI is constructed in this manner for each relevant substance. The last VoI, referred to as VoI ${ }^{\mathrm{Irrel}}$, is constructed containing all samples whose intensities do not correspond with any of the substances of interest. Evidently, the relevant substances can be selected depending on the goals of the specialist. In general, only the HB and LB are relevant, in which case the volumes $\mathrm{VoI}^{\mathrm{HB}}, \mathrm{VoI}^{\mathrm{LB}}$, and $\mathrm{VoI}^{\mathrm{Irrel}}$ are generated.

Each VoI is encoded independently, resulting in a single compressed file for each VoI. At the end of the encoding process, all compressed files are encapsulated in a single DICOM-compliant file. As described below, the original RV can be recovered losslessly if all VoIs are encoded and stored losslessly.

\subsection{RV Reallocation}

The original RV is reconstructed in the "RV Reallocation Procedure". If all the VoIs are decoded, such procedure is performed by adding the VoIs in a sample-bysample basis. In our previous example the original $\mathrm{RV}$ is recovered as $\mathrm{RV}_{x, y, z}=$ $\mathrm{VoI}_{x, y, z}^{\mathrm{HB}}+\mathrm{VoI}_{x, y, z}^{\mathrm{LB}}+\mathrm{VoI}_{x, y, z}^{\mathrm{Irrel}}$. As stated in the introduction, it may happen that only the $\mathrm{VoI}^{\mathrm{HB}}$ and/or the $\mathrm{VoI}^{\mathrm{LB}}$ are needed. Such a situation occurs when, for instance, the compressed images are stored in a server and the specialist wants to access the data from a remote location. In such a case, only the VoIs containing HB and LB are transmitted, reducing both the latency and the transmitted data. 


\subsection{Variable Offset}

The "RV Allocation by Substances" procedure may cause that the sample intensities within the VoIs contain numerical discontinuities. These discontinuities are produced by the zeroes corresponding to substances stored in other VoIs and may negatively affect the coding performance of the compression system. To partially tackle this issue, an offset operation is applied to all VoIs containing relevant substances. This operation subtracts the minimum $\mathrm{HU}$ value minus 1 for each $\mathrm{VoI}^{\mathrm{S}}$. The offset process is performed before coding and is inverted after decoding. No offset is employed during the encoding of VoI ${ }^{\text {Irrel }}$, so the corresponding codestream is decoded directly.

\section{Results and Discussion}

Extensive experimental tests have been carried out to evaluate different aspects of the proposed method, namely, the savings in the stored and/or transmitted data needed to compute the $3 \mathrm{D}$ morphometry without error, the computational time required for encoding and decoding the original RV and the VoIs, and the error incurred in the 3D morphometry when the VoIs are decompressed in a lossy regime. The results reported for our method are compared to those achieved when storing and/or transmitting the original RV conventionally (i.e., employing a single volume). The results are reported in terms of the amount of compressed data required for the reconstruction of the relevant samples, expressed in bits per sample (bps). It is computed as $\frac{N}{X Y Z}$, with $N$ denoting the number of bits needed to decompress the relevant VoI, and $\mathrm{X}, \mathrm{Y}$, and $\mathrm{Z}$ denoting its respective dimension.

The coding parameters employed for each coding system are selected to achieve maximum coding performance 1 The reset interval is set to 64 in the JPEG-LS standard. For JPEG2000, zero levels of reversible 5/3 wavelet transform and codeblocks of $64 \times$

\footnotetext{
${ }^{1}$ The employed implementations are found in: LZW - http://www.gzip.org

JPEG-LS - http://www.hpl.hp.com/research/info_theory/loco/

JPEG2000 - http://www.kakadusoftware.com/

H.264 - http://iphome.hhi.de/suehring/html
} 
64 are used. For H.264, the High 4:4:4 Predictive Profile in lossless mode is employed. We recall that this mode bypasses the transform and quantization. In order to comply with this profile, all frames are coded in the YUV 4:4:4 colour space.

\subsection{Lossless coding performance}

The first test evaluates the coding performance achieved when all data corresponding to the HB, LB, and other substances are stored losslessly. Table 3 reports the achieved results with the best method highlighted in blue. These results indicate that the bitrate needed to code the VoIs containing HB and/or LB is significantly lower than that needed to code the original RV, for all coding techniques tested. These results also indicate that H.264 is the best coding technique to encode the original RV and the irrelevant substances, while JPEG2000 obtains the highest coding performance for HB and LB. When using JPEG2000, the amount of data required to store HB and LB is reduced in $97.75 \%$ and $92.70 \%$, respectively, on average. The superior results achieved by H.264 for the coding of the original RV and the VoI ${ }^{\text {Irrel }}$ are due to the use of motion compensation to exploit inter-slice redundancies, which can not be exploited in HB and LB due to the discontinuities derived from the allocation procedure. Unfortunately, the slices coded using motion compensation via H.264 cannot be decoded independently, which may become a drawback when the 3D morphometry is computed. This issue does not come up with JPEG2000, which is the best option if scalability by substance is needed.

The last column of Table 3 provides an indication of the performance loss produced when coding the RV via three separate VoIs as compared to the coding of the original RV conventionally. This loss is on average $0.03,0.09,0.44$ and $0.65 \mathrm{bps}$, respectively, for JPEG2000, LZW, JPEG-LS, and H.264. This penalization is due to the extra headers needed to identify the multiple codestreams in our approach, and due to the discontinuities introduced during the allocation procedure. In the case of JPEG2000, this penalization is particularly small, though for the other coding systems it is slightly larger. 
Table 3: Evaluation of the lossless coding performance achieved with the proposed method when used with LZW, JPEG-LS, JPEG2000, and H.264. Results are reported in bps. Cells with a dash indicate that the corresponding biological substance does not appear in the RV. The first column of each method reports the coding bitrate when coding the original RV conventionally, whereas columns 2-4 report the results when coding different substances. The last column of each method reports the penalization incurred when coding the substances in different VoIs.

\begin{tabular}{|c|c|c|c|c|c|c||c|c|c|c|c|}
\hline RV Loc. & RV\# & RV & HB & LB & Irrel & $\begin{array}{c}\text { HB+LB } \\
+ \text { IIrel } \\
-\mathrm{RV}\end{array}$ & RV & HB & LB & Irrel & $\begin{array}{c}\text { HB+LB } \\
+ \text { Irrel } \\
-\mathrm{RV}\end{array}$ \\
\hline
\end{tabular}

\begin{tabular}{|c|c|c|c|c|c|c|c|c|c|c|c|}
\hline & & & & LZ & & & & & JPEG & & \\
\hline \multirow{6}{*}{ Hip 1} & 1 & 8.24 & 0.09 & 0.43 & 7.81 & +0.06 & 7.44 & 0.09 & 0.51 & 7.33 & +0.37 \\
\hline & 2 & 9.08 & 0.07 & 0.34 & 8.75 & +0.06 & 8.31 & 0.07 & 0.40 & 8.26 & +0.34 \\
\hline & 3 & 8.36 & 0.09 & 0.45 & 7.92 & +0.08 & 7.73 & 0.09 & 0.54 & 7.67 & +0.46 \\
\hline & 4 & 10.90 & 0.06 & 0.29 & 10.60 & +0.04 & 10.03 & 0.06 & 0.34 & 9.99 & +0.28 \\
\hline & 5 & 7.31 & 0.07 & 0.54 & 6.81 & +0.08 & 6.61 & 0.07 & 0.64 & 6.48 & +0.45 \\
\hline & 6 & 6.99 & 0.05 & 0.42 & 6.61 & +0.07 & 6.32 & 0.05 & 0.49 & 6.11 & +0.38 \\
\hline \multirow{6}{*}{ Head 1} & 1 & 4.22 & - & 0.05 & 4.18 & +0.02 & 3.58 & - & 0.06 & 3.58 & +0.06 \\
\hline & 2 & 9.98 & 0.34 & 0.93 & 8.80 & +0.07 & 8.70 & 0.30 & 0.90 & 8.02 & +0.32 \\
\hline & 3 & 6.20 & - & 0.42 & 5.84 & +0.06 & 5.18 & - & 0.39 & 5.01 & +0.19 \\
\hline & 4 & 9.60 & 0.04 & 0.72 & 8.90 & +0.05 & 8.74 & 0.04 & 0.68 & 8.32 & +0.22 \\
\hline & 5 & 8.60 & - & 0.10 & 8.53 & +0.03 & 7.75 & - & 0.12 & 7.75 & +0.11 \\
\hline & 6 & 8.81 & 0.02 & 0.61 & 8.23 & +0.03 & 8.68 & 0.02 & 0.58 & 8.32 & +0.45 \\
\hline Head 2 & 1 & 7.56 & 0.30 & 1.08 & 6.28 & +0.08 & 6.46 & 0.27 & 1.02 & 5.72 & +0.34 \\
\hline \multirow{6}{*}{ Head 3} & 1 & 7.38 & 0.70 & 1.85 & 5.17 & +0.27 & 6.40 & 0.71 & 2.17 & 5.26 & +1.23 \\
\hline & 2 & 9.38 & 0.55 & 1.58 & 7.55 & +0.24 & 8.56 & 0.58 & 1.95 & 7.59 & +1.09 \\
\hline & 3 & 9.69 & 0.55 & 1.19 & 8.16 & +0.17 & 8.62 & 0.54 & 1.47 & 7.98 & +0.98 \\
\hline & 4 & 9.88 & 0.12 & 0.62 & 9.25 & +0.07 & 8.98 & 0.12 & 0.71 & 8.71 & +0.41 \\
\hline & 5 & 11.36 & 0.23 & 0.91 & 10.40 & +0.11 & 10.50 & 0.25 & 1.13 & 10.07 & +0.65 \\
\hline & 6 & 7.69 & 0.23 & 0.70 & 6.86 & +0.08 & 8.47 & 0.23 & 0.85 & 7.62 & +0.10 \\
\hline \multicolumn{2}{|c|}{ Average } & 8.49 & 0.22 & 0.70 & 7.72 & +0.09 & 7.76 & 0.22 & 0.73 & 7.40 & +0.44 \\
\hline
\end{tabular}

\begin{tabular}{|c|c|c|c|c|c|c|c|c|c|c|c|}
\hline & & & & JPEG2 & & & & & H.26 & & \\
\hline \multirow{6}{*}{ Hip 1} & 1 & 7.73 & 0.08 & 0.38 & 7.36 & +0.03 & 6.12 & 0.12 & 0.52 & 6.07 & +0.47 \\
\hline & 2 & 8.82 & 0.06 & 0.30 & 8.53 & +0.03 & 6.76 & 0.10 & 0.42 & 6.69 & +0.37 \\
\hline & 3 & 8.13 & 0.07 & 0.41 & 7.75 & +0.04 & 6.23 & 0.11 & 0.57 & 6.19 & +0.53 \\
\hline & 4 & 10.37 & 0.05 & 0.26 & 10.13 & +0.02 & 8.08 & 0.08 & 0.35 & 8.04 & +0.32 \\
\hline & 5 & 7.04 & 0.06 & 0.48 & 6.61 & +0.04 & 5.50 & 0.09 & 0.64 & 5.36 & +0.50 \\
\hline & 6 & 6.67 & 0.04 & 0.37 & 6.42 & +0.04 & 5.27 & 0.07 & 0.51 & 5.14 & +0.39 \\
\hline \multirow{6}{*}{ Head 1} & 1 & 4.24 & - & 0.04 & 4.21 & +0.01 & 3.38 & - & 0.06 & 3.38 & +0.06 \\
\hline & 2 & 9.35 & 0.28 & 0.80 & 8.36 & +0.00 & 8.07 & 0.36 & 1.01 & 7.63 & +0.65 \\
\hline & 3 & 6.24 & - & 0.34 & 5.92 & +0.01 & 5.56 & - & 0.43 & 5.45 & +0.32 \\
\hline & 4 & 9.03 & 0.03 & 0.63 & 8.45 & -0.01 & 8.10 & 0.06 & 0.80 & 7.87 & +0.57 \\
\hline & 5 & 8.73 & - & 0.08 & 8.67 & +0.02 & 8.07 & - & 0.12 & 8.07 & +0.12 \\
\hline & 6 & 8.21 & 0.02 & 0.51 & 7.74 & -0.01 & 7.12 & 0.03 & 0.62 & 6.98 & +0.48 \\
\hline Head 2 & 1 & 6.96 & 0.25 & 0.94 & 5.87 & -0.01 & 6.05 & 0.33 & 1.13 & 5.48 & +0.61 \\
\hline \multirow{6}{*}{ Head 3} & 1 & 7.08 & 0.64 & 1.65 & 5.04 & +0.02 & 5.65 & 0.88 & 2.37 & 4.83 & +1.70 \\
\hline & 2 & 8.76 & 0.49 & 1.42 & 7.12 & +0.05 & 6.57 & 0.67 & 1.97 & 6.10 & +1.57 \\
\hline & 3 & 9.11 & 0.49 & 1.08 & 7.77 & +0.06 & 7.13 & 0.62 & 1.53 & 6.64 & +1.15 \\
\hline & 4 & 9.35 & 0.10 & 0.56 & 8.82 & +0.03 & 7.08 & 0.16 & 0.78 & 7.02 & +0.73 \\
\hline & 5 & 10.64 & 0.19 & 0.82 & 9.83 & +0.03 & 8.07 & 0.29 & 1.18 & 7.95 & +1.07 \\
\hline & 6 & 7.18 & 0.20 & 0.63 & 6.49 & +0.03 & 5.33 & 0.27 & 0.88 & 5.15 & +0.73 \\
\hline \multicolumn{2}{|c|}{ Average } & 8.44 & 0.19 & 0.62 & 7.72 & +0.03 & 6.56 & 0.26 & 0.84 & 6.34 & +0.65 \\
\hline
\end{tabular}

\subsection{Evaluation of the Variable Offset}

Table 4 reports the bitrate achieved when losslessly coding all data corresponding to the HB and LB using the proposed approach. In these results, the offset operation 
Table 4: Evaluation of the coding performance gains achieved by the use of the offset operation. Results are reported in bps, on average for all microCT images of the corpus. The savings provided by the offset is reported within parentheses.

\begin{tabular}{|c|c|c|}
\cline { 2 - 3 } \multicolumn{1}{c|}{} & HB & LB \\
\hline LZW & $0.22(0 \%)$ & $0.70(0 \%)$ \\
\hline JPEG-LS & $0.25(12 \%)$ & $0.79(8.5 \%)$ \\
\hline JPEG2000 & $0.21(9.5 \%)$ & $0.64(3 \%)$ \\
\hline H.264 & $0.29(10.5 \%)$ & $0.90(7 \%)$ \\
\hline
\end{tabular}

is not used. The improvement in bitrate provided by such operation is reported within the parentheses. As previously mentioned, the offset provides no improvement for encoding the original RV and/or the irrelevant VoI, so such cases are not reported in this table. Results suggest that the offset improves the coding performance of JPEGLS, JPEG2000 and H.264 for both HB and LB substances.

\subsection{Computational Time}

The following test assesses computational time. The results are reported separately for encoding, and for decoding. The "RV allocation procedure" is based on a threshold technique, which has negligible computational costs, so it is not considered in this evaluation. Only the results for JPEG2000 are reported due to its competitive performance. This experiment is conducted using the JPEG2000 Kakadu software v7.4 on an Intel Core i7 @ 3.4 GHz. 4 threads of execution are employed for encoding and decoding.

Figure 2 reports the execution time spent by our method as a percentage of the computational time spent by the conventional approach that codes the original RV. The fraction of zeroes within each volume is depicted with a red dot. Note that our method encodes 3 VoIs, each the same size as the original RV. This may seem to require three times the computational time of a conventional coder. However, the experimental results depicted in the figure show that the encoding and decoding times have an inverse relationship with the fraction of zeroes within the volume. The complexity of encoding $\mathrm{HB}+\mathrm{LB}+$ Irrel is approximately 2.4 times higher than the computational complexity 


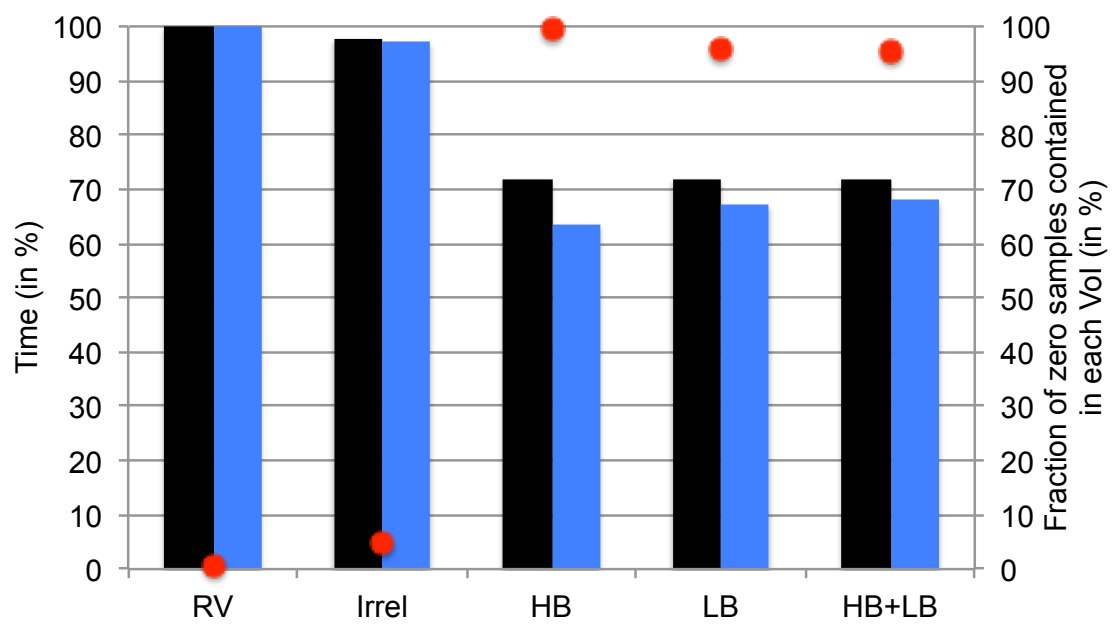

Figure 2: Evaluation of the computational time spent to code the original RV conventionally and for different VoIs. Black and blue bars show the encoding and decoding time, respectively. Orange circles denote the percentage of zeros of the different volumes.

of the conventional approach.

\subsection{Errors introduced in lossy regimes}

The last test compares the amount of data transmitted by the proposed and the conventional method to achieve a specific percentage of error in the 3D morphometry. Let $n$ denote the bitrate required by our approach to losslessly transmit $\mathrm{VoI}^{\mathrm{HB}}$ and $\mathrm{VoI}^{\mathrm{LB}}$. When the proposed method transmits $n$ bps, the 3D morphometry error for $\mathrm{VoI}^{\mathrm{HB}}$ and $\mathrm{VoI}^{\mathrm{LB}}$ is computed without error. Table 5 reports the percentage of error achieved in the $3 \mathrm{D}$ morphometry when the conventional approach transmits $n, 2 n$, $4 n, \ldots$ bps. These results are reported in average for all the RVs of the corpus. The 3D morphometry (i.e., $B V / T V, B S / B V$, $T b . T h, T b . N$, and $T b . S p$ ) is computed for the $\mathrm{HB}$ and LB, as it is commonly done, so the results are reported separately for each substance. The results of Table 5 suggest that to achieve an error lower than 18\%, 10\%, and $2 \%$ for the $\mathrm{HB}$, the conventional method needs to respectively transmit $4 n, 8 n$, and $32 n$ bps [25]. For the LB, these bitrates are $2 n, 8 n$, and $16 n$ bps. This indicates that the conventional method needs to transmit 32 and 16 times more data than the 
Table 5: 3D morphometry error (in \%) for HB and LB substances. The error is computed between the original and coded RVs at the specified bitrates.

\begin{tabular}{|l|c|c|c|c|c|c|c|c|c|c|}
\cline { 2 - 12 } \multicolumn{1}{c|}{} & \multicolumn{4}{c|}{ HB } & \multicolumn{4}{c|}{ LB } \\
\cline { 2 - 13 } & $V_{v}$ & $S_{v}$ & $T b . T h$ & $T b . N$ & $T b . S p$ & $V_{v}$ & $S_{v}$ & $T b . T h$ & $T b . N$ & $T b . S p$ \\
\hline$n$ bps & $42.35 \%$ & $36.19 \%$ & $26.20 \%$ & $57.67 \%$ & $36.19 \%$ & $19.19 \%$ & $11.26 \%$ & $11.67 \%$ & $34.31 \%$ & $11.26 \%$ \\
\hline $2 n$ bps & $23.09 \%$ & $20.63 \%$ & $16.87 \%$ & $36.24 \%$ & $20.63 \%$ & $6.46 \%$ & $4.75 \%$ & $4.67 \%$ & $11.77 \%$ & $4.75 \%$ \\
\hline $4 n$ bps & $15.06 \%$ & $12.66 \%$ & $11.07 \%$ & $24.60 \%$ & $12.66 \%$ & $0.55 \%$ & $4.80 \%$ & $5.06 \%$ & $4.46 \%$ & $4.80 \%$ \\
\hline $8 n$ bps & $6.27 \%$ & $6.92 \%$ & $6.40 \%$ & $12.34 \%$ & $6.92 \%$ & $0.08 \%$ & $2.09 \%$ & $2.14 \%$ & $2.06 \%$ & $2.09 \%$ \\
\hline $16 n \mathrm{bps}$ & $2.53 \%$ & $1.87 \%$ & $1.81 \%$ & $4.32 \%$ & $1.87 \%$ & $0.34 \%$ & $0.07 \%$ & $0.08 \%$ & $0.27 \%$ & $0.07 \%$ \\
\hline $32 n \mathrm{bps}$ & $1.70 \%$ & $1.41 \%$ & $1.37 \%$ & $3.07 \%$ & $1.41 \%$ & $0.00 \%$ & $0.00 \%$ & $0.00 \%$ & $0.00 \%$ & $0.00 \%$ \\
\hline
\end{tabular}

proposed method to achieve a negligible error in the 3D morphometry of the HB and $\mathrm{LB}$, respectively.

\section{Conclusions}

Preclinical micro-computed tomography (microCT) images are employed together with 3D morphometry evaluation for medical research. Typical region-of-interest coding techniques encode the relevant data and disregard the rest, which is not adequate in many medical environments. The method proposed in this paper uses a volumeof-interest coding technique that provides many interesting features for medical applications. First, it identifies the substances of the microCT through a thresholding segmentation technique based on the Hounsfield scale. Second, it allocates the substances in different VoIs, encoding them independently and with any coding system supported in DICOM. And third, it permits the extraction and transmission of only the relevant substances without needing to decode the whole image. Experimental results indicate that the proposed method reduces in more than $90 \%$ the stored and/or transmitted data when recovering specific substances of the image.

\section{Acknowledgment}

The authors thank the collaboration of the Center of Animal Biotechnology and Gene Therapy, and the Department of Animal Health and Anatomy, both from the 
Universitat Autònoma de Barcelona. Our special thanks to Marc Navarro and Jesús

Ruberte, who helped to validate and test the proposed method.

This work has been partially funded by FEDER, by the Spanish Government (MINECO), and by the Catalan Government, under Grants TIN2012-38102-C03-03, RYC-201005671, 2014 SGR 691, and BE-DGR 2012.

\section{References}

[1] G. R. Mundy, Metastasis: Metastasis to bone: causes, consequences and therapeutic opportunities, Nature Reviews Cancer 2 (8) (2002) 584 - 593.

[2] X. Chen, J. C. H. Goh, S. H. Teoh, S. D. De, R. Soong, T. Lee, Localized sclerotic bone response demonstrated reduced nanomechanical creep properties, ELSEVIER Journal of the Mechanical Behavior of Biomedical Materials 17 (0) (2013) $198-208$.

[3] J. Xia, L. Wang, Small-animal whole-body photoacoustic tomography: A review, Biomedical Engineering, IEEE Transactions on 61 (5) (2014) 1380 - 1389.

[4] T. Hildebrand, A. Laib, R. Müller, J. Dequeker, P. Rüegsegger, Direct threedimensional morphometric analysis of human cancellous bone: Microstructural data from spine, femur, iliac crest, and calcaneus, ASBMR Journal of Bone and Mineral Research 14 (7) (1999) 1167-1174.

[5] V. Fritz, P. Louis-Plence, F. Apparailly, D. Nol, R. Voide, A. Pillon, J.-C. Nicolas, Micro-CT combined with bioluminescence imaging: A dynamic approach to detect early tumorbone interaction in a tumor osteolysis murine model, ELSEVIER Bone 40 (4) (2007) 1032-1040.

[6] H. R. Buie, N. A. Bosma, C. M. Downey, F. R. Jirik, S. K. Boyd, Micro-CT evaluation of bone defects: Applications to osteolytic bone metastases, bone cysts, and fracture, ELSEVIER Medical Engineering \& Physics 35 (11) (2013) 1645-1650.

[7] H. R. Buie, G. M. Campbell, R. J. Klinck, J. A. MacNeil, S. K. Boyd, Automatic segmentation of cortical and trabecular compartments based on a dual threshold 
technique for in vivo micro-CT bone analysis, ELSEVIER Bone 41 (4) (2007) $505-515$.

[8] Digital Image and Communication in Medicine, DICOM (Aug. 2014).

URL http://medical.nema.org/

[9] X. Cao, H. Huang, Current status and future advances of digital radiography and PACS, IEEE Engineering in Medicine and Biology Magazine 19 (5) (2000) 8088.

[10] T. Welch, A technique for high-performance data compression, IEEE Computer 17 (6) (1984) 8-19.

[11] Information technology - digital compression and coding of continuous-tone still images - requirements and guidelines (Sep. 1992).

[12] Information technology lossless and near-lossless compression of continuoustone still images baseline (Jun. 1999).

[13] Information technology - JPEG 2000 image coding system - Part 1: Core coding system (Dec. 2000).

[14] T. Wiegand, G. Sullivan, G. Bjontegaard, A. Luthra, Overview of the H.264/AVC video coding standard, IEEE Transaction on Circuits and Systems for Video Technology 13 (7) (2003) 560-576.

[15] P. Schelkens, A. Munteanu, J. Barbarien, M. Galca, X. Giro-Nieto, J. Cornelis, Wavelet coding of volumetric medical datasets, IEEE Transactions on Medical Imaging 22 (3) (2003) $441-458$.

[16] Z. Xiong, X. Wu, S. Cheng, J. Hua, Lossy-to-lossless compression of medical volumetric data using three-dimensional integer wavelet transforms, IEEE Transactions on Medical Imaging 22 (3) (2003) 459-470.

[17] M. Penedo, W. Pearlman, P. Tahoces, M. Souto, J. Vidal, Region-Based wavelet coding methods for digital mammography, IEEE Transactions on Medical Imaging 22 (10) (2003) 1288-1296. 
[18] V. Sanchez, R. Abugharbieh, P. Nasiopoulos, Symmetry-based scalable lossless compression of 3D medical image data, IEEE Transactions on Medical Imaging 28 (7) (2009) $1062-1072$.

[19] J. Bartrina-Rapesta, F. Aulí-Llinàs, J. Serra-Sagristà, JPEG2000 ROI Coding Through Component Priority for Digital Mammography, ELSEVIER Computer Vision and Image Understanding (1) (2010) 59 - 68.

[20] V. Bairagi, A. Sapkal, ROI based DICOM Compression for Telemedicine, Vol. 38, SPRINGER Sadhana-Academy, 2013, pp. 123-131.

[21] General Electrics, eXplore Locus MicroCT (Feb. 2015). [link].

URL http://www3.gehealthcare.com/en/support/support_ documentation_library

[22] Y. Jiang, J. Zhao, E.-Y. Liao, R.-C. Dai, X.-P. Wu, H. K. Genant, Application of micro-CT assessment of 3-D bone microstructure in preclinical and clinical studies, SPRINGER Journal of Bone and Mineral Metabolism 23 (1) (2005) 122131.

[23] Doube M, Klosowski MM, Arganda-Carreras I, Cordelieres F, Dougherty RP, Jackson J, Schmid B, Hutchinson JR, Shefelbine SJ, BoneJ 1.3.9 (Jan. 2015). URL http: / / bonej.org/

[24] Wayne Rasband, ImageJ (Jan. 2015). URL http://rsbweb.nih.gov/ij/

[25] J. Gasser, P. Ingold, K. Grosios, A. Laib, S. Hämmerle, B. Koller, Noninvasive monitoring of changes in structural cancellous bone parameters with a novel prototype micro-CT, Journal of Bone and Mineral Metabolism 23 (2005) 90-96.

[26] Center for animal biotechnology and gene therapy (CBATEG), Universitat Autònoma de Barcelona (Mar. 2015). [link]

URL http: / / cbateg - uab.cat/ 
[27] M. L. Bouxsein, S. K. Boyd, B. A. Christiansen, R. E. Guldberg, K. J. Jepsen, R. Mller, Guidelines for assessment of bone microstructure in rodents using microcomputed tomography, Journal of Bone and Mineral Research 25 (7) (2010) 1468-1486. doi:10.1002/jbmr.141.

[28] H. Yang, K. D. Butz, D. Duffy, G. L. Niebur, E. A. Nauman, R. P. Main, Characterization of cancellous and cortical bone strain in the in vivo mouse tibial loading model using microCT-based finite element analysis, Bone 66 (2014) 131-139. doi:10.1016/j.bone.2014.05.019.

[29] M. J. Weinberger, G. Seroussi, G. Sapiro, The LOCO-I Lossless Image Compression Algorithm: Principles and Standardization into JPEG-LS, IEEE Transactions on Image Processing 9 (8) (2000) 1309-1324.

[30] P. Dong, S. Haupert, B. Hesse, M. Langer, P.-J. Gouttenoire, V. Bousson, F. Peyrin, 3D osteocyte lacunar morphometric properties and distributions in human femoral cortical bone using synchrotron radiation micro-ct images, ELSEVIER Bone 60 (0) (2014) 172 - 185. doi:http://dx.doi.org/10. $1016 / j$. bone.2013.12.008

\section{Biographies}

\section{Joan Bartrina-Rapesta}

received the M.S., and Ph.D. degrees in computer science from the Universitat Autnoma de Barcelona, Spain, in 2006, and 2009, respectively. He was awarded with a doctoral fellowship from Universitat Autònoma de Barcelona. He collaborated in the development a JPEG2000 Part 1 implementation. His research interests includes image and video coding, and interactive image and video transmission.

\section{Francesc Aulí-Llinàs}

is a Ramn y Cajal Fellow. He received the B.E. and Ph.D. degrees in computer science in 2002 and 2006, respectively, from the Universitat Autònoma Barcelona. Since 2002 
he has been funded in competitive fellowships. In 2013, he received a distinguished RLetter given by the IEEE Communications Society. His interests are image and video coding, computing, and transmission.

\section{Ian Blanes}

received the B.S., M.S., and Ph.D. in computer science from the Universitat Autònoma de Barcelona, in 2007, 2008, and 2010, respectively. In 2010, he was a visiting postdoctoral researcher at the Centre National d'Etudes Spatiales, France. He was the second-place finisher as best computer-science student of Spain by the Spanish Ministry of Education.

\section{Michael W. Marcellin}

graduated summa cum laude with the B.S. degree in Electrical Engineering from San Diego State University in 1983. He received the M.S. and Ph.D. degrees in Electrical Engineering from Texas A\&M University in 1985 and 1987, respectively. He is with the University of Arizona, where he holds the title of Regents' Professor of Electrical and Computer Engineering.

\section{Victor Sanchez, \\ XXXXXXX}

\section{Joan Serra-Sagristà,}

received the Ph.D. degree in computer science from Universitat Autònoma Barcelona (UAB), Spain, in 1999. His current research interests focus on image coding for remote sensing and telemedicine applications. He serves as Associate Editor of IEEE Trans. on Image Processing. He was the recipient of the Spanish Intensification Young Investigator Award in 2006. 